\title{
Severe Anemia in Children: A Clue of Primary Intestinal Lymphangectasia
}

\author{
Cecilia Mantegazza ${ }^{1 *}$, Erica Galli ${ }^{1}$, Dario Dilillo ${ }^{1}$, Luciano Maestri $^{2}$, Milena Meroni $^{2}$, Giovanna Riccipetitoni ${ }^{2}$, \\ Vincenzo Villanacci ${ }^{3}$ and Gian Vincenzo Zuccotti ${ }^{1}$ \\ ${ }^{1}$ Department of Pediatrics, Italy \\ ${ }^{2}$ Department of Pediatric Surgery, Italy
}

${ }^{3}$ Department of Pathological Anatomy, Italy

*Corresponding author: Cecilia Mantegazza, Department of Pediatrics, Italy

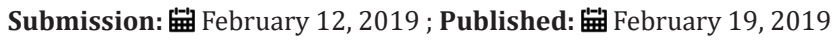

\begin{abstract}
Background: Primary intestinal lymphangiectasia is a rare disease described in adults and children. It usually presents itself as an excessive protein loss into the gastrointestinal tract due to stasis and rupture of limph vessels; however, few reports in adults and very few in children have described associated obscure gastrointestinal bleeding.

Case report: we report a case of a 1-year-old child who was referred to our hospital for anasarca, significant pallor and weakness. Laboratory tests showed severe iron-deficiency anemia, hypoalbuminemia and hypogammaglobulinemia. No proteinuria was found in his urinalysis. Fecal occult blood was highly positive. Investigations were not indicative of an enteropathy. During diagnostic work-up, a 99mT-DTPA scintigraphy showed an abnormal diffusion of the radioisotope in the small bowel. An intraoperative endoscopy and the histopathology examination of the entero-biopsy specimens of the small bowel were indicative of primary intestinal lymphangiectasia. After two blood and multiple albumin transfusions, the patient was first started on parenteral nutrition and afterwards on a beneficial low-fat, high protein diet and medium chain triglycerides supplementation and discharged.
\end{abstract}

Conclusion: The current case highlights the importance of considering abnormalities of the lymphatic system as a cause of severe anemia in children.

Keywords: Intestinal lymphangiectasia, Anemia, Protein losing enteropathy, Children

Abbreviations: PIL: Primary Intestinal Lymphangiectasia; GI: Gastrointestinal; MCT: Medium Chain Triglycerides; PLE: Protein Losing Enteropathy; IBD: Inflammatory Bowel Disease

\section{Case Presentation}

Primary intestinal lymphangiectasia (PIL) is a rare cause of obscure gastrointestinal (GI) bleeding that may range from chronic blood loss to massive bleeding. Few reports have been described in adults [1-4] and very few in children [5]. Here, we describe a case of an infant diagnosed with PIL who presented with severe iron deficiency anemia and hypoalbuminemia, in order to demonstrate the importance of considering abnormalities of the lymphatic system as a cause of anemia.

A 1 year-old child presented to Buzzi children hospital in October 2017 with 2 months of diffuse edema, pallor and weakness; his growth in weight was stable on the $10^{\text {th }}-25^{\text {th }}$ centile; otherwise his medical history was unremarkable. Physical examination revealed significant pallor and swelling with anasarca. In particular he presented with periorbital, hands, scrotal, sacral, feet and limb-pitting edema. Moreover, cardiac examination revealed high heart rate and an unknown systolic 2/6 murmur. Upon primary evaluation, laboratory tests showed severe irondeficiency anemia (hemoglobin 3,8g/dl, mean corpuscular volume $57,3 \mathrm{fl}$, ferritin $1 \mu \mathrm{g} / \mathrm{l}$, reticulocytes $0,9 \%$ ), hypoalbuminemia $(2 \mathrm{~g} /$ $\mathrm{dl})$ and hypogammaglobulinemia $(0,1 \mathrm{~g} / \mathrm{dl})$. No proteinuria was found in his urinalysis. Abdominal ultrasound revealed ascites; no alterations were found on chest X-ray or echocardiography. Fecal occult blood was highly positive.

In the hypothesis of an enteropathy we first considered celiac disease as a diagnosis, however specific celiac type serology was negative and upper gastrointestinal endoscopy and histology were unremarkable. On ileo-colonoscopy no alteration was found. A 99mT-DTPA scintigraphy showed an abnormal diffusion of the radioisotope in the intestinal lumen in particular in the small bowel and suggested a diagnosis of a protein losing enteropathy Therefore, 
an exploratory laparoscopy with intraoperative endoscopy of the small bowel was performed through a small enterotomy and an edematous aspect of the jejunal mucosa with diffuse multiple scattered white spots and swollen villi was found (Figure 1). Postoperative recovery was uneventful. The histopathology report of the entero-biopsy specimens revealed markedly dilated mucosal lymph vessels with no evidence of inflammation (Figure 2). Immunohistochemistry was negative for lymphoma. We therefore established the diagnosis of PIL. After two blood and multiple albumin transfusions, the patient was first started on parenteral nutrition and afterwards on a low-fat, high protein diet and medium chain triglycerides (MCT) supplementation and discharged. At follow-up, 1 month after the last blood and albumin transfusions, the patient appeared in well condition with no edema, his hemoglobin and albumin were $11,4 \mathrm{~g} / \mathrm{dl}$ and $4,3 \mathrm{~g} / \mathrm{dl}$ respectively.

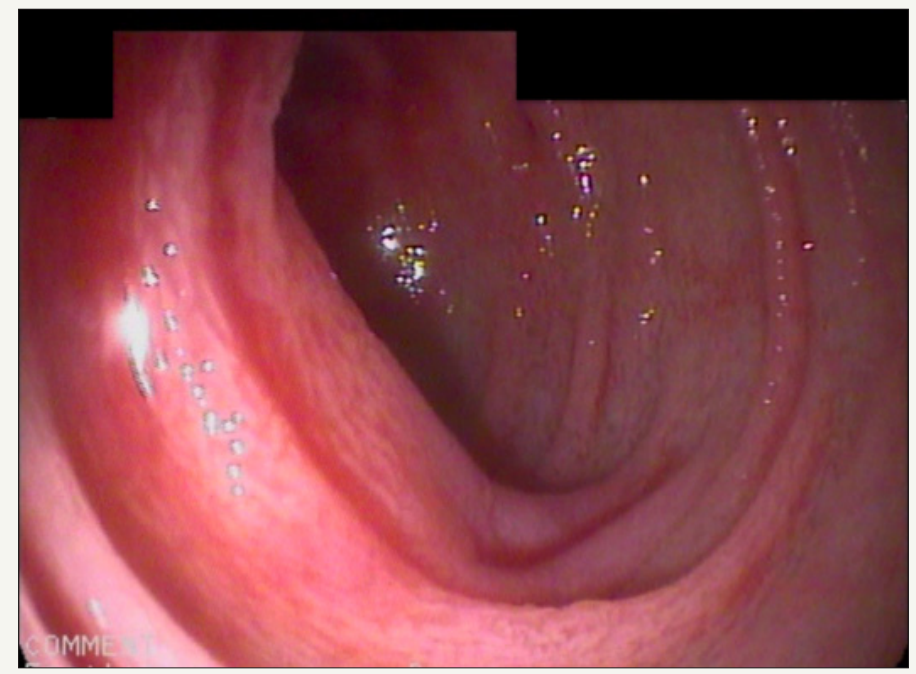

Figure 1: Jejunal endoscopic picture.

Note: Edematous aspect of mucosa with whitish, swollen villi.

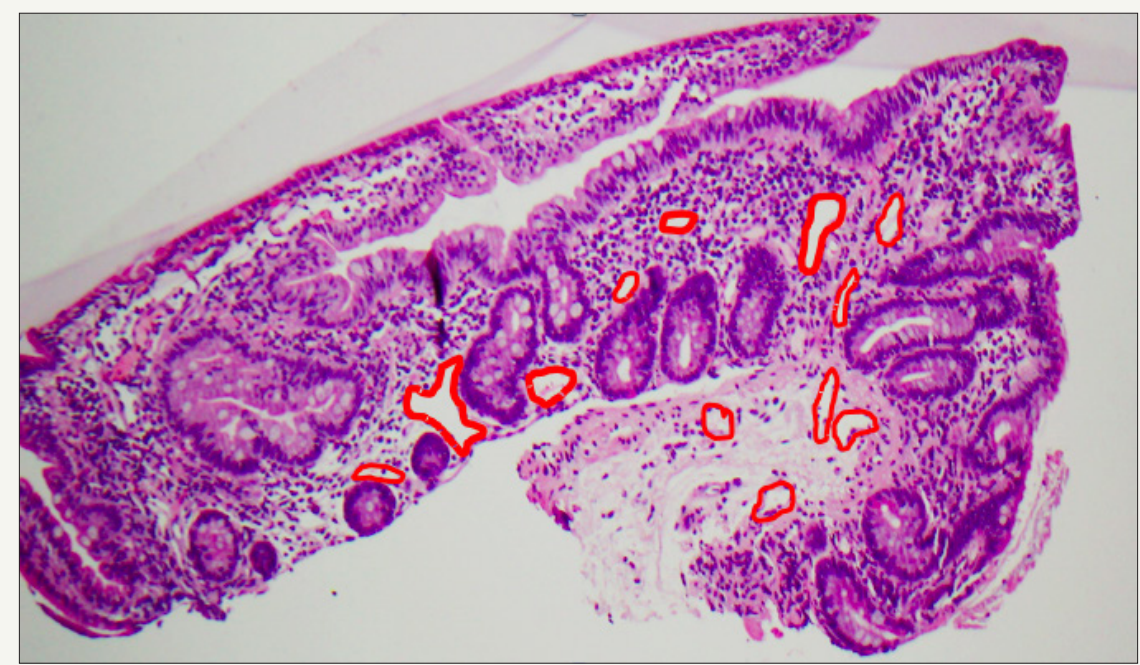

Figure 2: Jejunal biopsy.

Note: It is markedly dilated lymphatic ducts (highlighted in red).

Intestinal lymphangectasia is a rare condition characterized by diffuse or local dilatation of the enteric lymphatics, with subsequent stasis and rupture of the lymph vessels leading to leakage of protein-rich lymphatic fluid into the GI tract. It can be primary (congenital) or secondary to condition causing obstruction of the vessels or elevated lymph pressure as inflammatory bowel disease (IBD), lymphoma, congestive heart failure, constrictive pericarditis or intestinal malrotation [6].
PIL is generally diagnosed in the first years of life; its clinical presentation range from completely asymptomatic patients to a picture of severe protein losing enteropathy (PLE). However, PIL's clinical features usually include edema and GI symptoms like diarrhea, nausea or vomiting. Its main biochemical findings include hypoalbuminemia, hypogammaglobulinemia, hypocalcemia, hypocholesterolemia and lymphopenia. Iron deficiency anemia is normally not seen in patients with intestinal lymphangectasia. 
Howbeit our patient presented with a severe microcytic anemia and required multiple blood transfusions, leading initially the diagnosis towards a GI mucosal disease like inflammatory and ulcerative conditions (IBD, infections or GI malignancies) or malabsorption conditions (celiac disease, food induce enteropathy or eosinophilic gastroenteritis). However, these diagnoses were excluded. Of notice the child's albumin requirement was nevertheless prevalent, therefore a lymphatic disorder was considered. PIL anemia can be due to malabsorption or chronic blood loss from ulceration or coincident angiodysplasia. Although rare, massive GI bleeding can even occur [2-4]. An obstruction of the normal efferent chyle flow from the intestine that leads to a raising in intraluminal pressures and opening of latent lymphatic-venous or arterial connections, has been postulated to be the cause of anemia [4,7]. Indeed, a retrograde flow of blood into the lymphatics is therefore allowed and the rupture of the dilated lymphatics into the intestine may explain the GI bleeding. A permanent low-fat diet supplemented with MCT is the cornerstone of PIL medical treatment [8]. Its use, by normalizing the lymphatic intraluminal pressure, not only prevents the loss of albumin into the GI tract but also the bleeding. The dietary intervention was effective in our patient and both hemoglobin and albumin normalized during follow-up. However non-responders to the diet have been described in the literature; these patients may require thorough diagnostic work up to exclude an associated angiodysplasia and may benefit from other therapeutic options such as octreotide or tranexamic acid, angiography embolization or resection of the lesion.

\section{References}

1. Jha AK, Thandassery BR, Goenka KM (2014) Focal intestinal lymphangiectasia: an unusual cause of acute overt obscure gastrointestinal bleeding. J Dig Endoscopy 4(5): 162-163.

2. Baichi MM, Arifuddin RM, Mantry PS (2007) Acute gastrointestinal bleeding from focal duodenal lymphangiectasia. Scand J Gastroenterol 42(10): 1269-1270

3. Perisic VN, Kokai G (1991) Bleeding from duodenal lymphangiectasia. Arch Dis Child 66(1): 153-154.

4. Vignes S, Bellanger J (2008) Primary intestinal lymphangiectasia (Waldmann's disease). Orphanet J Rare Dis 22(3): 5.

5. Lom J, Dhere T, Obideen K (2010) Intestinal lymphangiectasia causing massive gastrointestinal bleed. J Clin Gastroenterol 44(1): 74-75.

6. Poirier VC, Alfidi RJ (1973) Intestinal lymphangiectasia associated with fatal gastrointestinal bleeding. Am J Dig Dis 18(1): 54-58.

7. Davidson JD, Flynn EP, Kirkpatrick JB (1966) Protein-losing enteropathy and intestinal bleeding. The role of lymphatic-venous connections. Ann Intern Med 64(3): 628-635.

8. Desai AP, Guvenc BH, Carachi R (2009) Evidence for medium chain triglycerides in the treatment of primary intestinal lymphangiectasia. Eur J Pediatr Surg 19(4): 241-245.
Creative Commons Attribution 4.0 International License

For possible submissions Click Here

\section{Submit Article}

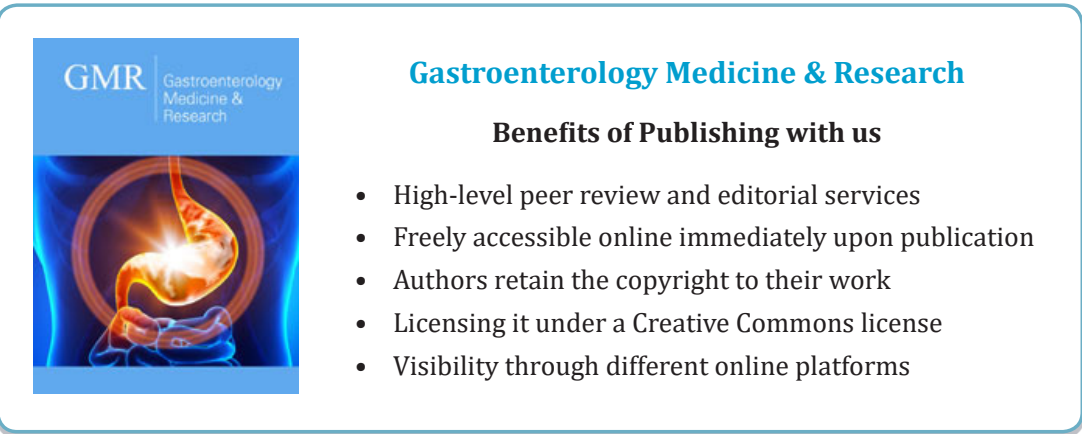

